Entgegen der Ansicht des Klägers kann ferner auch die Tatsache, daß die seiner strafgerichtlichen Verurteilung wegen öffentlicher Billigung eines Totschlags zugrunde liegenden Veröffentlichungen in der Bochumer Studentenzeitung bereits mehr als sieben Jahre zurückliegen, seinem Begehren nicht zum Erfolg zu verhelfen. $[\ldots]$

Es sind keine Anhaltspunkte dafür sichtbar geworden, daß der Kläger sich zuverlässig erkennbar von seinen früheren Ansichten und Zielen abgewandt hat. Davon abgesehen kommt es entscheidend auf die Eignungsmerkmale an, die zur Zeit der Entscheidung des Dienstherrn über die Bewerbung bestehen. (Vgl. BVerwG, Urteil vom 6. Februar i975, a. a. O., S. 339 f.) [...]

Die Revision ist nicht zuzulassen, weil die Voraussetzungen von $\$$ I32 Abs. 2 VwGO und von $\$ I 27 des Beamtenrechtsrahmengesetzes nicht gegeben sind. [Az.: VI A 9I/76; K I I88/74 Gelsenkirchen]

\title{
Anmerkung zu den vier vorstehenden Entscheidungen
}

$\mathrm{Zu}$ kommentieren sind drei Entscheidungen aus Bayern, die zu rechtsstaatlichen Hoffnungen Anlaß geben, und eine aus Preußen, die Illusionen über das Ausmaß rechtlich gesicherter "Freiheitlichkeit « in der "streitbaren « Republik vorbeugen soll. ${ }^{1}$ Die Auswahl ist weder repräsentativ noch wurde sie in polemischer Absicht vorgenommen. Immerhin mag das Ergebnis der ebenso landläufigen wie einfältigen Regionalisierung politischer Freiheit nach A- und B-Ländern entgegenwirken. »Es gibt noch Richter in Bayern «, merkte die Gewerkschaft Erziehung und Wissenschaft mit Erleichterung an. ${ }^{2}$ Wer die Verwaltungspraxis und die Argumentationsfiguren freistaatlicher Einstellungsbehörden studiert, wird zugeben, daß man hier besonders dringend einer Justiz bedarf, die den gesetzlich zuständigen oder selbsterklärten Verfassungsschutzagenturen rechtsstaatliche Lichter aufsteckt.

Trotz der prozessualen Unterschiede 3 bietet sich ein Vergleich der vier Entscheidungen an: In allen war die politische Treuepflicht von Bewerbern oder Angehörigen des öffentlichen Dienstes, genauer: des Volksschullehreramtes, der Bezugspunkt richterlicher Wahrheitsfindung. Alle Kläger bzw. Antragsteller waren gewerkschaftlich orientiert oder organisiert (GEW und IG Metall), darüber hinaus aber keine Mitglieder einer sich als kommunistisch oder sozialistisch verstehenden Organisation. Außerdem hatten die Gerichte jeweils eine im weitesten Sinne »linke« hochschulpolitische Vergangenheit zu würdigen, an der die Schulaufsichtsbehörden aufgrund verfassungsschützerischer Amtshilfe Anstoß genommen hatten. Bei ihren Verdächtigungen haben Regierung und Verwaltung auch das Absurde nicht gemieden: das unterlassene morgendliche Schulgebet, eine Reise nach Gera zur Friedrich-

\footnotetext{
I Wer sich der traditionellen preußischen Ausdeutung der politischen Treuepflicht vergewissern möchte, sei auf die Rechtsprechung des preußischen OVG verwiesen, insbesondere OVGE 77,493;78, 445, die auch das BVerfG in seinem "Radikalen «-Beschluß rezipiert - BVerfGE $39,334 \mathrm{ff} . / 362 \mathrm{ff}$

2 Die Demokratische Schule, s/1977, S. 2.

3 Vergleicht man die methodische und hermeneutische Sorgfalt, mit der das VG Augsburg im Verfahren nach $₫{ }_{12} 3$ VerGO vorgegangen ist, mit dem saloppen Berufungsurteil des OVG Münster, dann kann man nicht behaupten, der Rechtsstreit sei im ersten Fall summarisch, im zweiten Fall gründlich entschieden worden.
} 
Engels-Gedenkfeier, der rote Fahnenrest vor dem Haus, die Teilnahme an einer genehmigten Demonstration gegen das Bayerische Hochschulgesetz, ein Wahlbündnis mit SHB und MSB-Spartakus usw. wurden bemüht, um die Zweifel an der Verfassungstreue zu belegen. Gemeinsam ist allen gerichtlichen Entscheidungen, daß ihnen sog. "Anhörungs «- oder "Einstellungsgespräche" vorausgegangen waren, die nicht ernsthaft der Sachaufklärung dienten (und zur Selbstanfechtung führten), sondern als Rituale abliefen, die darauf angelegt waren, die einmal geäußerten Zweifel zu bestätigen. ${ }^{4}$

Darf man von den Regierungs- und Verwaltungsbehörden viel Sympathie für die administrative "Lösung " von Diskussionsprozessen und ein beträchtliches Maß an Unverständnis hinsichtlich der Notwendigkeit von Kritik erwarten, so wäre es immerhin wünschenswert, daß diese Behörden sich vor der Verhängung von Denkund Berufsverboten der Rechtsprechung des Bundesverfassungsgerichts vergewisserten, zumal sich besonders dessen konturenlose Ausdeutung der Treuepflicht von Beamten besonderer Beliebtheit erfreut.' Das BVerfG hat auch entschieden: "Die Verfassung schränkt zwar den Grundsatz politischer Toleranz um seiner Erhaltung willen ein, begegnet aber auch den Feinden der Freiheit, deren politische Betätigung sie beschneiden muß, nur mit rechtsstaatlichen Mitteln, soll sich die freiheitliche Demokratie nicht selbst untreu werden. " ${ }^{6}$ Auf eben diese Mittel legen die oben zitierten Entscheidungen - mit Ausnahme des Urteils des OVG Münster - die Einstellungsbehörden fest:

I. Sie präzisieren die formellen Voraussetzungen für die Ablehnung eines Bewerbers bzw. die Entlassung eines Angehörigen des öffentlichen Dienstes mangels Verfassungstreue. $\mathrm{Da}$ das Selbstverständliche längst nicht mehr selbstverständlich ist, dürfen wir als rechtsstaatlichen Fortschritt verbuchen, wenn Gerichte daran erinnern, daß

- »es sich bei der Feststellung der verfassungstreuen Haltung . . . um ein höchstpersönlich zu treffendes und zu verantwortendes, in die Zukunft gerichtetes Werturteil handelt" (VG Augsburg),

- bei der gerichtlichen Kontrolle ein strenger Maßstab anzulegen (und im streitigen Fall auch angelegt worden) ist,

- es bei der Uberprüfung der Verfassungstreue »allein auf die im Verhalten zutage getretene Gesamthaltung ankommt " (VGH München), also nicht auf ungewollte Auswirkungen oder auf eine unterstellte Gesinnung,

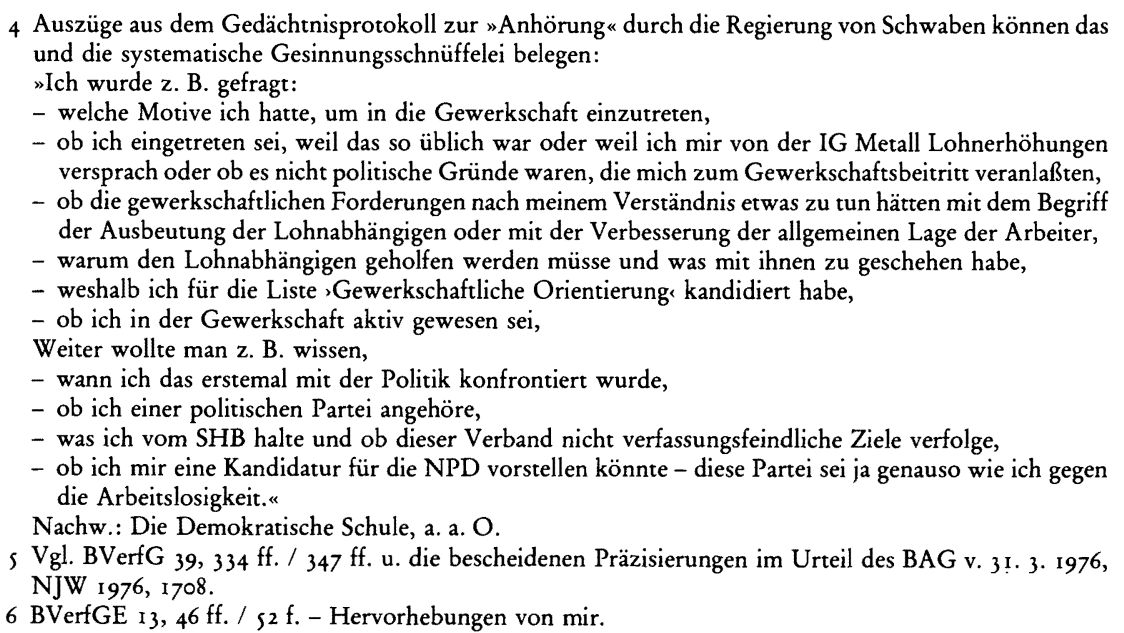


- die Entlassung oder Nichteinstellung nur mit "Gründen, die in der Person liegen« (VG Regensburg) gerechtfertigt werden kann, also nicht mit offiziellen Bedrohungsanalysen oder den Feindbildern und politischen Vorurteilen einer von Verschwörungsvorstellungen heimgesuchten Bürokratie,

- die allgemeinen Regeln über die materielle Beweislast damit wieder in Kraft treten: "da der Bewerber sich auf ein durch Art. 33 II GG verbürgtes Recht beruft, trägt die Behörde die materielle Beweislast für ihre dieses Recht belastende Behauptung der fehlenden Verfassungstreue « ${ }^{7}$

Instanzen, denen bei der Produktion von Demokratiefeinden vage Verdächtigungen leicht von der Hand gehen, die es mit rechtsstaatlichen Verfahrensgarantien so wenig genau nehmen, daß sie sich nicht immer an disziplinarrechtliche Vorschriften gebunden fühlen, geraten vor einer Justiz in Beweisnot, die sich anschickt nach den Grundsätzen: in dubio pro reo und in dubio pro libertate zu verfahren. Wiederum das OVG Münster ausnehmend (und zwar nicht erst wegen eines mißliebigen Ergebnisses, sondern bereits aufgrund der Art und Weise wie das Gericht seine Willkürkontrolle ${ }^{8}$ ausgeübt hat), haben die angerufenen Verwaltungsgerichte eine innerstaatliche Arbeitsteilung aufgekündigt, die sich in der wechselseitigen Beschaffung von Legitimität erschöpft.

2. Die Entscheidungen befassen sich in bemerkenswerter Weise auch mit den materiellen Voraussetzungen, unter denen Zweifel an der Verfassungstreue sich zu Einstellungshindernissen oder Entlassungsgründen verdichten.

Man tritt der Richterschaft nicht zu nahe, wenn man ihr generell ein Bedürfnis nach politischer Entlastung unterstellt, das ihnen von ihrer Ausbildung, ihrem Status und rechtssystematisch nahegelegt wird. Dieses Bedürfnis verfehlen Kläger und Anwälte, die dem Verwaltungsrichter einer Entscheidung über die Legalität des Weltkommunismus oder über die Illegalität des Imperialismus abverlangen. Schriftsätze, die darauf angelegt sind, enthalten ein fundamentales Mißverständnis des Verwaltungsprozesses. Er ist, man mag das bedauern, ein bürgerlicher Rechtsstreit. Keinen der Klageführer trifft hier der Vorwurf, eine falsche Prozeßstrategie verfolgt zu haben. Um so mehr überrascht die Bereitschaft der angerufenen Gerichte (Ausnahme OVG Münster), politische Fragen vergleichsweise extensiv inhaltlich zu untersuchen, um die Grenzmarken legaler politischer Praxis in der Bundesrepublik festzulegen. Vor dem Hintergrund der von der Exekutive betriebenen und von Teilen der Justiz nachvollzogenen Ausweitung und Verallgemeinerung der Verdachtsmomente gegen "Staats «- oder "Verfassungsfeinde «, d. h. der Einschränkung des Bereichs gesicherter politischer Freiheit, stimmt es zwar nicht euphorisch, aber es muß ermutigen, wenn Richter liberal-rechtsstaatliche Selbstverständlichkeiten respektieren:

- den engagierten Protest (zumindest den aus humanitären Gründen) gegen Flächenbombardements für legal halten (VGH München),

- gegen antifaschistisches Engagement keine Einwände haben (VGH München),

- eine gewerkschaftliche Orientierung nicht negativ sanktionieren, die sich über Wahl- und Zweckbündnisse mit kommunistischen Organisationen im Hochschulbereich durchsetzen will (VG Augsburg; VGH München),

- und erkennen, daß die rote Fahne Sinnbild auch meiner freiheitlich-demokratischen Idee" war und ist (VG Regensburg).

Die gerichtliche Würdigung der Beteiligung an (hochschul)politischen Bündnissen trägt bei zur Sicherung demokratischer Verfassungspositionen und zum Abbau

7 E. Martin, Extremistenbeschluß und demokratische Verfassung. In: Aus Politik und Zeitgeschichte/Beilage zur Wochenzeitschrift Das Parlament, B so/1973, S. 3 ff., 13.

8 Vgl. dazu BVerfGE 39, 354 u. 21,127 
politischer Berührungsängste. Dieser Beitrag sollte nicht deshalb unterschätzt werden, weil es sich hier nur um Einzelfälle handelt. Denn erstens sind diese Einzelfälle nicht untypisch gelagert; zweitens setzten Verdächtigungen und Berufsverbote in aller Regel an der individuellen Existenz an, um - spezialpräventiv - die individuelle politische Handlungsbereitschaft zu lähmen und - generalpräventiv - die Kritikund Konfliktfähigkeit politischer Organisationen auszuhöhlen. Im Kampf gegen die Auffächerung der Verdachtsmomente, die zu amtlichen Zweifeln an der Verfassungstreue und zur Ablehnung von Bewerbern für den öffentlichen Dienst führen - z. B. der Vorwurf, man selbst oder ein Angehöriger (Vater) gehöre einer, laut Verfassungsschutzbericht, »kommunistischen Tarnorganisation « $\mathrm{an}^{9}$ oder habe mit einer solchen zusammengearbeitet oder sei deren "Sympathisant « oder "sympathisiere « allgemein mit terroristischen Gewalttätern oder habe ein Untermietverhältnis mit einer Frau gehabt, die mit einem Mann verlobt war, der der Beteiligung an terroristischen Gewalttaten angeklagt (und, was keine Erwähnung fand, freigesprochen) wurde. ${ }^{10}$

Im Kampf gegen die systematische Verunsicherung der politischen Handlungsspielräume gegen Selbstzensur und Freiheitsverzicht kann auch eine Justiz wertvolle Hilfe leisten, die die Exekutive auf das Legalitätsprinzip und auf den Respekt vor Freiheitsrechten festlegt.

Die Entscheidungen des VGH München und der VGe Augsburg und Regensburg heben sich wohltuend $\mathrm{ab}$ von dem Spruch des OVG Münster. Hier schlägt das Bedürfnis nach politischer Entlastung um in einen Verzicht auf eine wenigstens formaljuristisch schlüssige Argumentation. Die knappe, etwa 4 Seiten lange Urteilsbegründung zeigt, wie man mit geringen Mitteln nichts (Überzeugendes) erreicht. Wer zwischen marxistischer Erkenntnistheorie und marxistisch-leninistischer Revolutionstheorie nicht zu unterscheiden vermag, die "Lehren von Marx und Lenin" pauschal für unvereinbar mit »unserer freiheitlichen demokratischen Grundordnung « hält, muß zwangsläufig zu dem Schluß kommen: »allein die Tatsache, daß der Kläger eingeräumt hat, er fühle sich dem Gedankengut des Marxismus-Leninismus verbunden, (war) geeignet, die bestehenden $Z$ weifel daran, daß er jederzeit für die freiheitliche demokratische Grundordnung eintrete, zu bestätigen. Bezeichnet sich ein Beamtenbewerber selbst als 'Marxist-Leninist, so bekennt er sich damit zu den Lehren von Marx und Lenin.....

Hatte das OVG Berlin sich noch bemüßigt gesehen, eine "Grenze zwischen wissenschaftlicher Theorie und politischem Ziel« zu ziehen, und den durch Art. 5 Abs. 3 GG geschützten Freiheitsraum dort enden zu lassen, "wo wissenschaftliche Erkenntnisse in die politische Wirklichkeit umgesetzt werden sollen und zu politischem Handeln aufgerufen wird ${ }^{11}{ }^{11}$ so erspart sich das OVG Münster die Frage nach der Reichweite der einschlägigen Art. 5 Abs. I, 4 Abs. I und 3 Abs. 3 GG im Vorbereitungsdienst für Volksschullehrer. Nachdem es sich souverän von verfassungsrechtlichen Uberlegungen dispensiert hat, sieht es am Ende - wen wundert's? - keinen Grund für die Zulassung der Revision. Eine Revision hat der VI. Senat des OVG Münster selbst vorgenommen: bei der Interpretation des Eignungskriteriums in Art. 33 II GG stellten die Richter nicht auf die fachliche Kompetenz und

9 Vgl. B. Engelmann, Trotz alledem. Deutsche Radikale 1777 - 1977. München 1977, S. 376.

Io So geschehen an der Universität Augsburg, die sich aufgrund einer entsprechenden Erkenntnis des Verfassungsschutzes weigerte, mit dem Wissenschaftler H. Bullens einen Arbeitsvertrag abzuschließen und sich erst nach Interventionen des Personalrates, der OTV und der GEW und nach kritischen Pressekommentaren dazu bereit fand, festzustellen, die Zweifel an der Verfassungstreue hätten sich als "nicht hinlänglich begründet « erwiesen. Nachw. Südd. Zeitung v. is./16. 10. 1977.

I I OVG Berlin v. I0. 6. I976. In: KJ I/1977, S. 76 ff. m. Anm. von B. v. Greiff und J. Perels. 
Bereitschaft zur Gesetzes- und Verfassungstreue ab, sondern auf die Gesinnung. Das im Verlauf der Entstehungsgeschichte des Art. 33 II GG gestrichene Beiwort "charakterlich " vor "Eignung " wird interpretativ gleichsam interpoliert. ${ }^{12}$ Im Namen der "Wehrhaftigkeit « des »Gesinnungsstaates « operiert das Gericht mit einer verdeckten Illoyalitätsvermutung, die in apodiktischen Aussagen zutage tritt, wie: "Bereits der der Verurteilung des Klägers ... zugrundeliegende Sachverhalt läßt eine Einstellung erkennbar werden, die Zweifel an seiner Verfassungstreue rechtfertigt.« oder: "hierzu bedarf es keiner weiteren Ausführungen« oder: ». . . allein die Tatsache, daß der Kläger eingeräumt hat, er fühle sich dem Gedankengut des Marxismus-Leninismus verbunden, (war) geeignet, die bestehenden Zweifel . . zu bestätigen «.

Gegen das OVG Münster »besteht Veranlassung zu bemerken, daß nicht schon ein Bekenntnis zum Marxismus eine Illoyalitätsvermutung begründet. Vor allem gegenüber Simplifikationen, wie sie der politischen Tagesdebatte üblich geworden sind, ist an die außerordentliche Spannweite heutiger Marxismen und Neomarxismen ${ }^{13}$ und die Prinzipien pluralistischer Offenheit und Toleranz zu erinnern. Das OVG Münster kennt im Unterschied zum BVerfG ${ }^{\mathrm{I} 4}$ und auch zum VG Augsburg weder historische noch biographische "Tiefe«. Für eine Straftat, auch wenn sie mehr als sieben Jahre zurückliegt ${ }^{15}$, verlangt das Gericht eine nachträgliche Distanzierung. Wer wie der Kläger sein Verhalten zu rechtfertigen versucht, gibt zu erkennen, »daß er sich nicht etwa in der Zwischenzeit von seiner früheren Einstellung und den von ihm verfolgten Zielen abgewandt hat «. Daß eine Behörde und ein Gericht "jedem Bewerber zugute halten $m u \beta$, daß Jugendliche oftmals erst nach vielen vorausgegangenen Fehlentscheidungen den rechten Weg zur Enwicklung und Reifung ihrer Persönlichkeit finden« (VG Augsburg), ist ein dem VI. Senat des OVG Münster offensichtlich fremder Gedanke. Die Forderung, man müsse früheren politischen Einstellungen und Aktionen eine nachträgliche Absage erteilen, beruht auf einem ebenso bornierten wie verbreiteten Verständnis der individuellen politischen Sozialisation. Diese wird nicht als kontinuierlicher und krisenhafter Prozeß politischen Lernens begriffen, sondern als notwendige schrittweise Anpassung an die herrschenden Normen eingefordert. Abweichendes Verhalten, gar die Auflehnung gegen die herrschenden Ideologien und die "guten politischen Sitten « bedarf daher einer ausdrücklichen Distanzierung. Eine frühere politische Identität muß nachträglich, vom Standpunkt des auf den »normalen Freiheitsgebrauch « eingeschworenen Staatsbürgers, diffamiert werden - ein Standpunkt, den in Deutschland traditionell die exekutive Staatsgewalt umreißt. Eine Selbstbezichtigung wie z. B. »Ich war dummerweise Kommunist « oder "Wie konnte ich nur gegen Vietnam demonstrieren? « signalisiert denjenigen, die sich aus Angst, karrierefördernder Vorsicht oder sonstigen Gründen angepaßt haben und nun zensierend die politische Tugend der Gesellschaft bewachen, daß da jemand seinen politischen Erfahrungszusammenhang $\mathrm{zu}$ blockieren bereit ist oder schon blockiert hat und mit ihrer eigenen Entwicklung sympathisiert, und daß die Formierung seines politischen Bewußtseins eine staatsverträgliche Inanspruchnahme seiner Grundrechte und ein »gemütliches Knecht-

I 2 So schon BVerwGE 47, 330. Kritisch dazu: J. Esser, Bemerkungen zur Unentbehrlichkeit des juristischen Handwerkzeugs, Jz 1975, S. 555 ff./557. Vgl. auch R. Dreier, Verfassung und Ideologie - Bemerkungen zum Radikalenproblem. In: Gedächtnisschrift für Friedrich Klein, Hrsg. v. D. Wilke und H Weber, München 1977, S. $86 \mathrm{ff} . / 107 \mathrm{f}$.

I 3 R. Dreier, ebd. S. I08; vgl. H. H. Holz, Strömungen und Tendenzen im Neomarxismus, München 1972.

14 Vgl. die "liberale Passage« des "Radikalen «-Beschlusses, BVerfGE 39, $356 \mathrm{f}$.

Is Der Tatbestand enthält keine näheren Angaben über die Art und Weise, in der der Kläger (beim OVG Münster) seinerzeit die Ermordnung des Grafen Spreti billigte. 
schaftsverhältnis« zu den ehedem kritisierten gesellschaftlichen Verhältnissen erwarten läßt. Ihm darf man den Zugang zur Bundeslade, will sagen: zum öffentlichen Dienst, gewähren. Einen solchen Anpassungsdruck, jedenfalls einen $Z$ wang zur Verstellung ${ }^{16}$, mag das VG Augsburg, insofern liberaler als das BVerfG, nicht ohne weiteres sanktionieren. Es verlangt weder den politischen Offenbarungseid vor dem Eintritt in den öffentlichen Dienst noch das Bekenntnis zur "politischen oder juristischen Tagesmeinung « noch die pauschale Ablehnung und Distanzierung von politischen und rechtlichen Anschauungen, die man nicht teilt. Der verbeamtete oder zu verbeamtende Staatsdiener muß lediglich einen »deutlichen Trennungsstrich ... gegenüber jedweden Auffassungen ziehen, die für ihn erkennbar einen Angriff gegen die freiheitliche demokratische Grundordnung enthalten «. Das Gericht widersetzt sich damit der tendenziellen Zerstörung politischen Denkens und politischer Identität in der Bundesrepublik durch die von Staats wegen inszenierten rituellen Beschwörungen der Loyalität zum System und durch die Aufherrschung des offiziellen Freund-Feind-Schemas auf den demokratischen Prozeß, dessen Resultat Abgrenzungs- und Unvereinbarkeitsbeschlüsse und Distanzierungen von dieser oder jener politischen Organisation und Strategie sind. ${ }^{17}$

Bei ihrer Kontrolle der exekutiven Produktion von "Staats «-, »Verfassungs«- und "Demokratiefeinden" vollstreckt die Rechtsprechung also nicht immer und nicht ohne weiteres aus den offiziellen Bedrohungsanalysen, wie sie die Verfassungsschutzberichte enthalten. Auch gibt sie der Abwehr von McCarthy-Methoden Flankenschutz, indem sie an einer grundsätzlichen Loyalitätsvermutung bei allen Bewerbern und Beamten festhält, die nicht Mitglieder einer für verfassungswidrig erklärten Organisation oder Partei sind; was praktisch auf die zutreffende Uberlegung hinausläuft, daß ein freiheitliche Demokratie in der Lage sein muß, die rechtsstaatlichen Folgekosten politischer Freiheit und pluralistischer Toleranz zu tragen. ${ }^{18}$ Dennoch oder vielleicht gerade deshalb muß eine insofern progressive Rechtsprechung eine "Vorwärtssperre errichten: sozialistische oder kommunistische Perspektiven liegen als organisierte Handlungsprogramme offensichtlich jenseits der Grenzen ihrer politischen Belastbarkeit und ihres Demokratieverständnisses. Die Mitgliedschaft in einer "Demokratischen Front« oder die Beteiligung an einem politischen Bündnis wird daher, soweit möglich, als individuelle politische Praxis qualifiziert. Eine politische Praxis, die die Gesellschaft als ganze kritisch ins Auge faßt, wird, soweit möglich, als "Stellungnahme zu Tagesfragen« oder zu speziellen Problemen (Hochschulgesetzgebung, Vietnam usw.), als »humanitärer

I6 Vgl. dazu: W. D. Narr, Anhörung, in: Kursbuch Juni 1975, u. ders., Verliert den Verstand nicht! - Anmerkungen zu einem Verhör, in: KJ 2/1975, S. I60 zur Problematik der Verstellung.

I7 Exemplarisch die Erklärung, die zwölf niedersächsischen Hochschullehrern zur Unterschrift vorgelegt wurde, die an der Herausgabe der Dokumentation "Buback - Nachruf « beteiligt waren "Im Zusammenhang mit den Ermittlungen der niedersächsischen Landesregierung zur Herausgabe der Dokumentation >Buback - ein Nachruf, erkläre ich:

Mord und Entführung oder überhaupt den Einsatz von Gewalt lehne ich in unserem freiheitlichen demokratischen Rechtsstaat unter jeder Bedingung ab. Deshalb verurteile ich terroristische Handlungen und alle Versuche, diese zu rechtfertigen.

Ich bin mir bewußt, daß ich als Beamter eine besondere Treupflicht gegenüber dem Staat habe. Diese fordert mehr als nur eine formal korrekte, im übrigen uninteressierte, kühle, innerlich distanzierte Haltung gegenüber Staat und Verfassung; sie fordert vom Beamten insbesondere, daß er sich eindeutig von Gruppen und Bestrebungen distanziert, die diesen Staat, seine verfassungsmäßigen Organe und die geltende Verfassungsordnung angreifen, bekämpfen und diffamieren. Ich werde meiner politischen Treuepflicht nachkommen. Diese hat sich insbesondere in Krisenzeiten und in ernsthaften Konfliktsituationen zu bewähren, in denen der Staat darauf angewiesen ist, daß der Beamte Partei für ihn ergreift. Ich distanziere mich in aller From von dem Verfasser und dem Inhalt des sogenannten Buback-Nachrufs.« Zitat nach FR v. 26. 10. 77.

I8 Vgl. R. Dreier, a. a. O., Anm. I3. 
Protest « kleingearbeitet. Zweckbündnisse erscheinen akzeptabel, soweit sie nicht auf eine Identifikation mit dem organisierten Kommunismus hindeuten. Alle politischen Aktionen haben vor dieser Justiz eine Legalisierungschance, wenn sie sich als "Jugendsünde« verstehen lassen. Damit nähert sich die Rechtsprechung Benedetto Croce, der dafür hält, daß wir bis zum zwanzigsten Lebensjahr unbedingt Kommunisten gewesen sein müssen, soll man uns nicht für herzlos halten, es aber mit dreißig nicht mehr sein dürfen, wollen wir nicht als unverständig gelten. Triumph einer liberalen, aber eben bürgerlichen Vernunft!

Günter Frankenberg

\section{Disziplinarverfahren im Zusammenhang mit einer Gedenkminute in einer Berliner Referendararbeitsge- meinschaft}

\section{EINLEITUNG DISZIPLINARISCHER VORERMITTLUNGEN}

Der Präsident des Kammergerichts

Berlin 19 (Charlottenburg), den 26.5. 77

- M 6II2 E KG -

$[\ldots]$

Betr.: Disziplinarverfahren

Sehr geehrter Herr M.!

Wegen des Vorfalls vom I 3. April 1977 in der Arbeitsgemeinschaft S I/66 - Sitzenbleiben während der Gedenkminute zu Ehren des ermordeten Generalbundesanwaltes Buback und seiner Mitarbeiter - führe ich gegen Sie nach \27 LDO Vorermittlungen. [...]

Hochachungsvoll

Dr. Dehnicke

\section{STELLUNGNAHME DER PERSONALVERSAMMLUNG DER BERLI- NER GERICHTSREFERENDARE*}

Am I 3. April 1977 wurden der ermordete Generalbundesanwalt Buback und seine beiden Begleiter in Form eines Staatsbegräbnisses beigesetzt. Einige Tage zuvor hatten hohe Berliner Politiker an die Berliner Bevölkerung appelliert, der Erschossenen an diesem Tage um 10.30 Uhr eine Minute lang schweigend zu gedenken.

Am gleichen Tag fanden für einige Berliner Gerichtsreferendare Pflichtarbeitsgemeinschaften statt. Gegen 10.30 Uhr an diesem Tag forderte der Leiter einer dieser Arbeitsgemeinschaften die Gerichtsreferendare auf, sich zum ehrenden Gedenken der durch das Attentat Ermordeten zu erheben.

Eine Kollegin und zwei Kollegen folgten dieser Aufforderung nicht, sie blieben während der Gedenkminute schweigend sitzen. Für den Präsidenten des Kammergerichts war dies Anlaß genug, gegen die Kollegin und die Kollegen Disziplinarver-

* Die Stellungnahme sollte als Anzeige im Berliner "Tagesspiegel« erscheinen, der "Tagesspiegel « lehnte es jedoch ab, die Anzeige zu veröffentlichen. 